\title{
Analysis of electro-visco-elastic contact problem with friction
}

\author{
A. Bachmar, T. Serrar* \\ Applied Mathematics Laboratory, Setif 1 University, 19000, Algeria.
}

\begin{abstract}
A quasistatic frictional contact problem is studied. The material behavior is modeled with a nonlinear electro-visco-elastic constitutive law, allowing piezoelectric effects. The body may come into contact with a rigid obstacle. Contact is described with the Signorini condition, a version of Coulomb's law of dry friction, and a regularized electrical conductivity condition. We derive a variational formulation of the problem, then, under a smallness assumption on the coefficient of friction, we prove an existence and uniqueness result of a weak solution for the model. The proof is based on arguments of elliptic variational inequalities and fixed points of operators. (C)2016 All rights reserved.
\end{abstract}

Keywords: Piezoelectric, frictional contact, electro-visco-elastic, fixed point, quasistatic process, Coulomb's friction law, variational inequality.

2010 MSC: 74M10, 74M15, 74F15, 49J40.

\section{Introduction}

The piezoelectric effect is the apparition of electric charges on surfaces of particular crystals after deformation. Its reverse effect consists of the generation of stress and strain in crystals under the action of the electric field on the boundary. A deformable material which presents such a behavior is called a piezoelectric material. Piezoelectric materials are used extensively as switches and actuary in many engineering systems.

\footnotetext{
${ }^{*}$ Corresponding author

Email addresses: Aziza_bechmar@yahoo.fr (A. Bachmar), serrartouffik@yahoo.fr (T. Serrar)
} 
Only some materials exhibit sufficient piezoelectricity are useful in applications. These include quartz, Rochelle salt, lead titanate zirconate ceramics, barium titanate, and polyvinylidene fluoride (a polymer film), and are used extensively as switches and actuators in many engineering systems, in radioelectronics, electroacoustics and in measuring equipment. We find general models for electroelastic materials in [6, 7] and more recently, in [1, 4, 8]. A static and a slip-dependent frictional contact problems for electro-elastic materials were studied in [2, 5] and in [9], respectively. A contact problem with normal compliance for electro-visco-elastic materials was investigated in [10]. In [11] and [12], we find the variational formulations of the corresponding problems, existence and uniqueness of weak solutions.

The novelty of the present paper is to extend the result when the contact and friction are modeled by Signorini's conditions and a nonlocal Coulomb's friction law, respectively. Moreover, the material behavior is assumed to be electro-visco-elastic.

The paper is structured as follows. In Section 2 we present the electro-visco-elastic contact model with friction and provide comments on the contact boundary conditions. In Section 3 we list the assumptions on the data and derive the variational formulation. In Section 4, we present our main existence and uniqueness results, which state the unique weak solvability of the Signorini's contact electro-visco-elastic problem with nonlocal Coulomb's friction law conditions.

\section{Problem statement}

We consider a body made of a piezoelectric material which occupies the domain $\Omega \subset \mathbb{R}^{d}(d \leq 3)$ with a Lipschitz boundary $\Gamma$. The body is modeled with an electro-visco-elastic constitutive law, allowing piezoelectric effects. Let $[0, T]$ be the time interval where $T>0$, and let $\Gamma$ be split into three measurable parts $\Gamma_{1}, \Gamma_{2}$ and $\Gamma_{3}$ such that meas $\left(\Gamma_{1}\right)>0$. We assume that the body is fixed on $\Gamma_{1}$ and surface tractions of density $h$ act on $\Gamma_{2}$. On $\Gamma_{3}$, the body may come into contact with a rigid obstacle. In other hand, $\Gamma$ splits into two measurable sets $\Gamma_{a}$ and $\Gamma_{b}$ such that meas $\left(\Gamma_{b}\right)>0$ and $\Gamma_{3} \subset \Gamma_{b}$. We assume that the electrical potential $q_{0}$ acts on $\Gamma_{a}$ and a surface electric charge of density $q_{2}$ acts on $\Gamma_{b}$, we assume that the problem is quasistatic. The piezoelectric effect is the apparition of electric charges on surfaces of particular crystals after deformation. We denote by $\mathbb{S}^{d}$ the space of second order symmetric tensors on the space $\mathbb{R}^{d}$ and use $\cdot$ and $|\cdot|$ for the inner product and the Euclidean norm on the space $\mathbb{R}^{d}$ (respectively, $\mathbb{S}^{d}$ ). Also $\nu$ represents the unit outward normal on $\Gamma$. The classical formulation of the electro-visco-elastic contact friction problem is described by:

Problem 2.1. $P$ : Find a displacement field $u: \Omega \times[0, T] \rightarrow \mathbb{R}^{d}$, a stress field $\sigma: \Omega \times[0, T] \rightarrow \mathbb{S}^{d}$, an electric potential field $\varphi: \Omega \times[0, T] \rightarrow \mathbb{R}$, and an electric displacement field $D: \Omega \times[0, T] \rightarrow \mathbb{R}^{d}$ such that

$$
\begin{aligned}
\sigma & =\mathcal{A} \varepsilon(\dot{u})+\mathcal{G} \varepsilon(u)-\xi^{*} E(\varphi) \text { in } \Omega \times[0, T], \\
D & =\beta E(\varphi)+\xi \varepsilon(u) \text { in } \Omega \times[0, T], \\
D i v \sigma+f_{0} & =0 \text { in } \Omega \times[0, T], \\
\operatorname{div} D & =q_{0} \text { in } \Omega \times[0, T], \\
u & =0 \text { on } \Gamma_{1} \times[0, T], \\
\sigma_{\nu} & =h \text { on } \Gamma_{2} \times[0, T], \\
u_{\nu} \leq 0, \sigma_{\nu} \leq 0, u_{\nu} \sigma_{\nu} & =0 \text { on } \Gamma_{3} \times[0, T], \\
\left\{\begin{array}{l}
\left|\sigma_{\tau}\right| \leq \mu p\left|R \sigma_{\nu}\right|, \\
\left|\sigma_{\tau}\right|<\mu p\left|R \sigma_{\nu}\right| \Longrightarrow \dot{u}_{\tau}=0,
\end{array}\right. & \text { on } \Gamma_{3} \times[0,
\end{aligned}
$$




$$
\begin{aligned}
\varphi & =0 \text { on } \Gamma_{a} \times[0, T], \\
D \nu & =q_{2} \text { on } \Gamma_{b} \times[0, T], \\
u(0) & =u_{0} \text { in } \Omega \times[0, T],
\end{aligned}
$$

where 2.1) and 2.2 are the electro-visco-elastic constitutive law of the material, we denote $\varepsilon(u)$ (respectively, $\left.E(\varphi)=-\nabla \varphi, \mathcal{A}, \mathcal{G}, \xi, \xi^{*}, \beta\right)$ the linearized strain tensor (respectively, electric field, the viscosity nonlinear tensor, the elasticity tensor, the third order piezoelectric tensor, and its transpose, the electric permittivity tensor), (2.3) and (2.4) represent the equilibrium equation, we mention that Divo, divD are the divergence operators, (2.5) and (2.6) are the displacement and traction boundary conditions, and (2.7) and (2.8) the Signorini's contact with a nonlocal Coulomb's friction law conditions. $u_{\nu}$ and $u_{\tau}$ (respectively, $\sigma_{\nu}$ and $\sigma_{\tau}$ ) denote the normal displacement and the tangential displacement (respectively, the normal stress and the tangential stress).

$R$ will represent a normal regularization operator that is a linear and continuous operator $R$ : $H^{-\frac{1}{2}}(\Gamma) \rightarrow L^{2}(\Gamma)$. We shall need it to regularize the normal trace of the stress which is too rough on $\Gamma . p$ is a non-negative function, the so-called friction bound, $\mu \geq 0$ is the coefficient of friction. The friction law was used in some studies with $p(r)=r_{+}$where $r_{+}=\max \{0, r\}$. Recently, from thermodynamic considerations, a new version of Coulomb's law is proposed, it consists to take

$$
p(r)=r(1-\alpha r)_{+},
$$

where $\alpha$ is a small positive coefficient related to the hardness and the wear of the contact surface. Equations (2.9) and (2.10) represent the electric boundary conditions. Finally, in (2.11) $u_{0}$ is the given initial displacement.

\section{Variational formulation and preliminaries}

For a weak formulation of the problem, first we introduce some notation. The indices $i, j, k, l$ range from 1 to $d$ and summation over repeated indices is implied. An index that follows a comma represents the partial derivative with respect to the corresponding component of the spatial variable, e.g., $u_{i, j}=\frac{\partial u_{i}}{\partial x_{j}}$. We also use the following notations

$$
\begin{aligned}
H & =\mathbb{L}^{2}(\Omega)^{d}=\left\{u=\left(u_{i}\right) / u_{i} \in \mathbb{L}^{2}(\Omega)\right\}, \\
\mathcal{H} & =\left\{\sigma=\left(\sigma_{i j}\right) / \sigma_{i j}=\sigma_{j i} \in \mathbb{L}^{2}(\Omega)\right\}, \\
H_{1} & =\left\{u=\left(u_{i}\right) / \varepsilon(u) \in \mathcal{H}\right\}, \\
\mathcal{H}_{1} & =\{\sigma \in \mathcal{H} / \operatorname{Div} \sigma \in H\} .
\end{aligned}
$$

The operators of deformation $\varepsilon$ and divergence Div are defined by

$$
\varepsilon(u)=\left(\varepsilon_{i j}(u)\right), \quad \varepsilon_{i j}(u)=\frac{1}{2}\left(u_{i, j}+u_{j, i}\right), \quad \operatorname{Div} \sigma=\left(\sigma_{i j, j}\right) .
$$

The spaces $H, \mathcal{H}, H_{1}$, and $\mathcal{H}_{1}$ are real Hilbert spaces endowed with the canonical inner products given by

$$
\begin{aligned}
(u, v)_{H} & =\int_{\Omega} u_{i} v_{i} d x, \quad \forall u, v \in H, \\
(\sigma, \tau)_{\mathcal{H}} & =\int_{\Omega} \sigma_{i j} \tau_{i j} d x, \quad \forall \sigma, \tau \in \mathcal{H}, \\
(u, v)_{H_{1}} & =(u, v)_{H}+(\varepsilon(u), \varepsilon(v))_{\mathcal{H}}, \quad \forall u, v \in H_{1}, \\
(\sigma, \tau)_{\mathcal{H}_{1}} & =(\sigma, \tau)_{\mathcal{H}}+(\operatorname{Div} \sigma, \text { Div } \tau)_{H}, \quad \forall \sigma, \tau \in \mathcal{H}_{1} .
\end{aligned}
$$


We denote by $|\cdot|_{H}$ (respectively, $|\cdot|_{\mathcal{H}},|\cdot|_{H_{1}}$, and $|\cdot|_{\mathcal{H} 1}$ ) the associated norm on the space $H$ (respectively, $\mathcal{H}, H_{1}$ and $\mathcal{H}_{1}$ ).

We use standard notations for the $\mathbb{L}^{p}$ and the Sobolev spaces associated with $\Omega$ and $\Gamma$, for a function $\psi \in H^{1}(\Omega)$ we still write $\psi$ to denote its trace on $\Gamma$ and $C$ is a generic positive constants whose value may change from line to line. We recall that the summation convention is applied to a repeated index.

For the electric displacement field we use two Hilbert spaces

$$
\mathcal{W}=\mathbb{L}^{2}(\Omega)^{d}, \quad \mathcal{W}_{1}=\left\{D \in \mathcal{W}, \operatorname{div} D \in \mathbb{L}^{2}(\Omega)\right\} .
$$

Endowed with the inner products

$$
(D, E)_{\mathcal{W}}=\int_{\Omega} D_{i} E_{i} d x, \quad(D, E)_{\mathcal{W}_{1}}=(D, E)_{\mathcal{W}}+(\operatorname{div} D, \operatorname{div} E)_{\mathbb{L}^{2}(\Omega)}
$$

And the associated norm $|\cdot|_{\mathcal{W}}$ (respectively, $|\cdot|_{\mathcal{W}_{1}}$ ). The electric potential field is to be found in

$$
W=\left\{\psi \in H^{1}(\Omega), \psi=0 \text { on } \Gamma_{a}\right\} .
$$

Since meas $\left(\Gamma_{a}\right)>0$, the following Friedrichs-Poincaré's inequality holds, thus

$$
|\nabla \psi|_{\mathcal{W}} \geq c_{F}|\psi|_{H^{1}(\Omega)}, \quad \forall \psi \in W
$$

where $c_{F}>0$ is a constant which depends only on $\Omega$ and $\Gamma_{a}$. On $W$, we use the inner product given by

$$
(\varphi, \psi)_{W}=(\nabla \varphi, \nabla \psi)_{\mathcal{W}}
$$

and let $|\cdot|_{W}$ be the associated norm. It follows from (3.1) that $|\cdot|_{H^{1}(\Omega)}$ and $|\cdot|_{W}$ are equivalent norms on $W$ and therefore $\left(W,|\cdot|_{W}\right)$ is a real Hilbert space.

Moreover, by the Sobolev trace Theorem, there exists a constant $\widetilde{c}_{0}$, depending only on $\Omega, \Gamma_{a}$ and $\Gamma_{3}$ such that

$$
|\psi|_{\mathbb{L}^{2}\left(\Gamma_{3}\right)} \leq \widetilde{c}_{0}|\psi|_{W}, \quad \forall \psi \in W .
$$

We recall that when $D \in \mathcal{W}_{1}$ is a sufficiently regular function, the Green's type formula holds

$$
(D, \nabla \psi)_{\mathcal{W}}+(\operatorname{div} D, \psi)_{\mathbb{L}^{2}(\Omega)}=\int_{\Gamma} D \nu \cdot \psi d a
$$

When $\sigma$ is a regular function, the following Green's type formula holds

$$
(\sigma, \varepsilon(v))_{\mathcal{H}}+(D i v \sigma, v)_{H}=\int_{\Gamma} \sigma \nu \cdot v d a, \quad \forall v \in H_{1} .
$$

Next, we define the space

$$
V=\left\{u \in H_{1}(\Omega) / u=0 \text { on } \Gamma_{1}\right\} .
$$

Since meas $\left(\Gamma_{1}\right)>0$, the following Korn's inequality holds

$$
|\varepsilon(u)|_{\mathcal{H}} \geq c_{K}|v|_{H_{1}}, \quad \forall v \in V
$$

where $c_{K}>0$ is a constant which depends only on $\Omega$ and $\Gamma_{1}$. On the space $V$ we use the inner product

$$
(u, v)_{V}=(\varepsilon(u), \varepsilon(v))_{\mathcal{H}},
$$


let $|\cdot|_{V}$ be the associated norm. It follows by $(3.2)$ that the norms $|\cdot|_{H_{1}(\Omega)}$ and $|\cdot|_{V}$ are equivalent norms on $V$ and therefore, $\left(V,|\cdot|_{V}\right)$ is a real Hilbert space. Moreover, by the Sobolev trace Theorem, there exists a constant $c_{0}$ depending only on the domain $\Omega, \Gamma_{1}$, and $\Gamma_{3}$ such that

$$
|v|_{\mathbb{L}^{2}\left(\Gamma_{3}\right)^{d}} \leq c_{0}|v|_{V}, \quad \forall v \in V .
$$

Finally, for a real Banach space $\left(X,|\cdot|_{X}\right)$ we use the usual notation for the space $\mathbb{L}^{p}(0, T ; X)$ and $W^{k \cdot p}(0, T ; X)$, where $1 \leq p \leq \infty, k=1,2, \ldots$ We also denote by $C(0, T ; X)$ and $C^{1}(0, T ; X)$ the spaces of continuous and continuously differentiable functions on $[0, T]$ with values in $X$, with the respective norms

$$
\begin{aligned}
|x|_{C(0, T ; X)} & =\max _{t \in[0, T]}|x(t)|_{X}, \\
|x|_{C^{1}(0, T ; X)} & =\max _{t \in[0, T]}|x(t)|_{X}+\max _{t \in[0, T]}|\dot{x}(t)|_{X} .
\end{aligned}
$$

In what follows, we assume the following assumptions on the problem $P$.

The viscosity operator $\mathcal{A}: \Omega \times \mathbb{S}^{d} \rightarrow \mathbb{S}^{d}$ satisfies

$$
\left\{\begin{array}{l}
(a): \mathcal{A}: \Omega \times \mathbb{S}^{d} \rightarrow \mathbb{S}^{d}, \\
(b): \exists M_{\mathcal{A}}>0 \text { such that: }\left|\mathcal{A}\left(x, \varepsilon_{1}\right)-\mathcal{A}\left(x, \varepsilon_{2}\right)\right| \leq M_{\mathcal{A}}\left|\varepsilon_{1}-\varepsilon_{2}\right| \\
\forall \varepsilon_{1}, \varepsilon_{2} \in \mathbb{S}^{d} \text {, a.e., } x \in \Omega, \\
(c): \exists m_{\mathcal{A}}>0 \text { such that: }\left|\mathcal{A}\left(x, \varepsilon_{1}\right)-\mathcal{A}\left(x, \varepsilon_{2}\right), \varepsilon_{1}-\varepsilon_{2}\right| \geq m_{\mathcal{A}}\left|\varepsilon_{1}-\varepsilon_{2}\right|^{2} \\
\forall \varepsilon_{1}, \varepsilon_{2} \in \mathbb{S}^{d}, \text { a.e., } x \in \Omega, \\
(d): \text { the mapping } x \rightarrow \mathcal{A}(x, \varepsilon) \text { is lebesgue measurable in } \Omega \text { for all } \varepsilon \in \mathbb{S}^{d}, \\
(e): \text { the mapping } x \rightarrow \mathcal{A}(x, 0) \in \mathcal{H} .
\end{array}\right.
$$

The elasticity operator $\mathcal{G}: \Omega \times \mathbb{S}^{d} \rightarrow \mathbb{S}^{d}$, satisfies

$$
\left\{\begin{array}{c}
(a): \mathcal{G}: \Omega \times \mathbb{S}^{d} \rightarrow \mathbb{S}^{d}, \\
(b): \exists M_{\mathcal{G}}>0 \text { such that: }\left|\mathcal{G}\left(x, \xi_{1}\right)-\mathcal{G}\left(x, \xi_{2}\right)\right| \leq M_{\mathcal{G}}\left|\xi_{1}-\xi_{2}\right| \\
\forall \xi_{1}, \xi_{2} \in \mathbb{S}^{d}, \text { a.e., } x \in \Omega, \\
(d): \text { the mapping } x \rightarrow \mathcal{G}(x, \xi) \text { is lebesgue measurable in } \Omega \text { for all } \xi \in \mathbb{S}^{d}, \\
(e): \text { the mapping } x \rightarrow \mathcal{G}(x, 0) \in \mathcal{H} .
\end{array}\right.
$$

The piezoelectric operator $\xi=\left(e_{i j k}\right): \Omega \times \mathbb{S}^{d} \rightarrow \mathbb{R}^{d}$, satisfies

$$
\left\{\begin{array}{l}
(a): \xi=\left(e_{i j k}\right): \Omega \times \mathbb{S}^{d} \rightarrow \mathbb{R}^{d}, \\
(b): \xi(x, \tau)=\left(e_{i j k}(x) \tau_{j k}\right) \forall \tau=\left(\tau_{i j}\right) \in \mathbb{S}^{d}, \text { a.e., } x \in \Omega, \\
(c): e_{i j k}=e_{i k j} \in \mathbb{L}^{\infty}(\Omega) .
\end{array}\right.
$$

The electric permittivity operator $\beta=\left(\beta_{i j}\right): \Omega \times \mathbb{R}^{d} \rightarrow \mathbb{R}^{d}$, satisfies

$$
\left\{\begin{array}{l}
(a): \beta=\left(\beta_{i j}\right): \Omega \times \mathbb{R}^{d} \rightarrow \mathbb{R}^{d}, \\
(b): \beta(x, E)=\left(b_{i j}(x) E_{j}\right) \forall E=\left(E_{i}\right) \in \mathbb{R}^{d} \text {, a.e., } x \in \Omega, \\
(c): b_{i j}=b_{j i} \in \mathbb{L}^{\infty}(\Omega), \\
(d): \exists m_{\beta}>0 \text { such that: } b_{i j}(x) E_{i} E_{j} \geq m_{\beta}|E|^{2} \forall E=\left(E_{i}\right) \in \mathbb{R}^{d}, x \in \Omega .
\end{array}\right.
$$

From the assumptions (3.6) and (3.7), we deduce that the piezoelectric operator $\xi$ (respectively, the electric permittivity operator $\beta$ ) is linear, has measurable bounded component denoted by $e_{i j k}$ (respectively, $b_{i j}$ ) and moreover, $\beta$ is symmetric and positive definite. 
Recall also that the transposed operator $\xi^{*}$ is given by $\xi^{*}=\left(e_{i j k}^{*}\right)$ where $e_{i j k}^{*}=e_{k i j}$ and the following equality holds

$$
\xi \sigma . v=\sigma . \xi^{*} v \quad \forall \sigma \in \mathbb{S}^{d}, \quad v \in \mathbb{R}^{d} .
$$

The friction function satisfies

$$
\left\{\begin{aligned}
& p: \Gamma_{3} \times \mathbb{R} \rightarrow \mathbb{R}_{+} \text {verifies } \\
&(a): \exists M>0 \text { such that: }\left|p\left(x, r_{1}\right)-p\left(x, r_{2}\right)\right| \leq M\left|r_{1}-r_{2}\right| \\
& \quad \text { for every } r_{1}, r_{2} \in \mathbb{R}, \text { a.e., } x \in \Gamma_{3}, \\
&(b): \text { the mapping }: x \rightarrow p(x, r) \text { is measurable on } \Gamma_{3}, \text { for every } r \in \mathbb{R}, \\
&(c): p(x, 0)=0, \text { a.e., } x \in \Gamma_{3} .
\end{aligned}\right.
$$

We note that 3.8 is satisfied in the case in which $p$ is given by 2.12 .

We also assume that the body forces and surface tractions have the regularity

$$
f_{0} \in \mathbb{L}^{2}(0, T ; H), \quad h \in \mathbb{L}^{2}\left(0, T ; \mathbb{L}^{2}\left(\Gamma_{2}\right)^{d}\right),
$$

as well as the densities of electric charges satisfy

$$
q_{0} \in \mathbb{L}^{2}\left(0, T ; \mathbb{L}^{2}(\Omega)\right), \quad q_{2} \in \mathbb{L}^{2}\left(0, T ; \mathbb{L}^{2}\left(\Gamma_{b}\right)\right) .
$$

We define the function $f:[0, T] \rightarrow V$ and $q:[0, T] \rightarrow W$ by

$$
\begin{aligned}
& (f(t), v)_{V}=\int_{\Omega} f_{0}(t) v d x+\int_{\Gamma_{2}} h(t) v d a, \quad \forall v \in V, t \in[0, T], \\
& (q(t), \psi)_{W}=-\int_{\Omega} q_{0}(t) \psi d x+\int_{\Gamma_{b}} q_{2}(t) \psi d a, \quad \forall \psi \in W, t \in[0, T]
\end{aligned}
$$

for all $u, v \in V, \psi \in W$ and $t \in[0, T]$, and note that conditions $(3.9)$ and (3.10) imply that

$$
f \in \mathbb{L}^{2}(0, T ; V), \quad q \in \mathbb{L}^{2}(0, T ; W),
$$

while the friction coefficient $\mu$ satisfies

$$
\begin{aligned}
\mu & \in \mathbb{L}^{\infty}\left(\Gamma_{3}\right), \mu(x) \geq 0, \text { a.e., on } \Gamma_{3}, \\
u_{0} & \in V .
\end{aligned}
$$

We denote by $K$ the subset of admissible displacements fields given by

$$
K=\left\{v \in H_{1} / v=0 \text { on } \Gamma_{1}, v_{\nu} \leq 0 \text { on } \Gamma_{3}\right\} .
$$

Let $K$ be a nonempty, closed and convex set of $V$.

We denote by $j$ the friction functional $j: \mathcal{H}_{1} \times K \rightarrow \mathbb{R}$

$$
j(\sigma, v)=\int_{\Gamma_{3}} \mu p\left|R \sigma_{\nu}\right|\left|v_{\tau}\right| d a .
$$

By a standard procedure based on Green's formula, we obtain the following formulation of the mechanical problem 2.1)-2.11.

Problem 3.1. PV : Find a displacement field $u: \Omega \times[0, T] \rightarrow \mathbb{R}^{d}$, a stress field $\sigma: \Omega \times[0, T] \rightarrow \mathbb{S}^{d}$, an electric potential field $\varphi: \Omega \times[0, T] \rightarrow \mathbb{R}$ and an electric displacement field $D: \Omega \times[0, T] \rightarrow \mathbb{R}^{d}$ such that

$$
\begin{aligned}
(\sigma(t), \varepsilon(w-\dot{u}(t)))_{\mathcal{H}}+j(\sigma, w)-j(\sigma, \dot{u}(t)) & \geq(f(t), w-\dot{u}(t)), & & \forall u, w \in V, \\
(D(t), \nabla \psi)_{\mathbb{L}^{2}(\Omega)^{d}}+(q(t), \psi)_{W} & =0, & & \forall \psi \in W, \\
u(0) & =u_{0} . & &
\end{aligned}
$$




\section{Existence and uniqueness result}

Our main result which states the unique solvability of Problem 3.1, is the following:

Theorem 4.1. Assume that (3.4)-(3.10), (3.13), and (3.14) hold, then the Problem 3.1 has a unique solution $(u, \varphi)$. Moreover, the solution satisfies

$$
\begin{aligned}
u & \in W^{1.2}(0, T ; K), \\
\varphi & \in W^{1.2}(0, T ; W), \\
\sigma & \in \mathbb{L}^{2}\left(0, T ; \mathcal{H}_{1}\right), \\
D & \in W^{1.2}\left(0, T ; \mathcal{W}_{1}\right) .
\end{aligned}
$$

We conclude that under the assumptions $(3.4)-(3.10),(3.13)$, and $(3.14)$, the mechanical problem (2.1)-(2.11) has a unique weak solution with the regularity (4.1)-(4.4).

The proof of this theorem will be carried out in several steps. It is based on arguments of elliptic variational inequalities (see [3]) and fixed point arguments.

let $G \in \mathbb{L}^{2}\left(0, T ; \mathcal{H}_{1}\right)$ and $\eta \in \mathbb{L}^{2}\left(0, T ; V^{\prime}\right)$ be given, we deduce a variational formulation of Problem $P V$.

Problem 4.2. $P V_{G \eta}$ : Find a displacement field $u_{G \eta}:[0, T] \rightarrow K$ such that

$$
\begin{aligned}
& \left\{\begin{array}{l}
u_{G \eta}(t) \in K\left(\mathcal{A} \varepsilon\left(\dot{u}_{G \eta}(t)\right), \varepsilon\left(w-\dot{u}_{G \eta}(t)\right)_{\mathcal{H}}+\left(\eta, w-\dot{u}_{G \eta}(t)\right)_{V^{\prime} \times V}\right. \\
+j(G, w)-j\left(G, \dot{u}_{G \eta}(t)\right) \geq\left(f(t), w-\dot{u}_{G \eta}(t)\right), \quad \forall w \in K
\end{array}\right. \\
& u_{G \eta}(0)=u(0) .
\end{aligned}
$$

We define $f_{\eta}(t) \in V^{\prime}$ for a.e., $t \in[0, T]$ by

$$
\left(f_{\eta}(t), w\right)_{V^{\prime} \times V}=(f(t)-\eta(t), w)_{V^{\prime} \times V}, \quad \forall w \in V .
$$

From (3.12), we deduce that

$$
f_{\eta} \in \mathbb{L}^{2}\left(0, T ; V^{\prime}\right)
$$

We define the operator $A: V \rightarrow V^{\prime}$ by

$$
(A v, w)_{V^{\prime} \times V}=(\mathcal{A} \varepsilon(v), \varepsilon(w))_{\mathcal{H}}, \quad \forall v, w \in V .
$$

We consider the following variational inequality.

Problem 4.3. $P V 1_{G \eta}$ : Find a displacement field $v_{G \eta}: \Omega \times[0, T] \rightarrow K$, such that

$$
\left.\left(A v_{G \eta}(t)\right), w-v_{G \eta}(t)\right)_{V^{\prime} \times V}+j(G, w)-j\left(G, v_{G \eta}(t) \geq\left(f_{\eta}(t), w-v_{G \eta}(t)\right)_{V^{\prime} \times V}, \quad \forall w \in K .\right.
$$

In the study of Problem 4.3, we have the following result.

Lemma 4.4. For all $G \in \mathbb{L}^{2}\left(0, T ; \mathcal{H}_{1}\right)$ and $\eta \in \mathbb{L}^{2}\left(0, T ; V^{\prime}\right)$, $P V 1_{G \eta}$ has a unique solution with the regularity

$$
v_{G \eta} \in \mathbb{L}^{2}(0, T ; K) .
$$


For all $u, v \in V, t \in[0, T]$, assumptions (3.4) imply that the operator $A$ is strongly monotone and Lipschitz continuous.

We can easily check that $j$ is convex lower semicontinuous and proper.

It follows from classical results for elliptic variational inequalities (see [3]) that there exists a unique $v_{G \eta} \in \mathbb{L}^{2}(0, T ; K)$.

Let now $u_{G \eta}:[0, T] \rightarrow K$ be a function defined by

$$
u_{G \eta}(t)=\int_{0}^{t} v_{G \eta}(s) d s+u_{0}, \quad \forall t \in[0, T] .
$$

In the study of Problem 4.2, we have the following result.

Lemma 4.5. Problem 4.2 has a unique solution satisfying the regularity expressed in (4.1).

Proof. The proof of Lemma 4.5 is a consequence of Lemma 4.4 and the relation (4.7).

In the second step, we use the displacement field $u_{G \eta}$ obtained in Lemma 4.5 to consider the following variational problem.

Problem 4.6. $P V 2_{G \eta}$ : Find an electric potential field $\varphi_{G \eta}: \Omega \times[0, T] \rightarrow W$ such that

$$
\left(\beta \nabla \varphi_{G \eta}(t), \nabla \psi\right)_{\mathbb{L}^{2}(\Omega)^{d}}-\left(\xi \varepsilon\left(u_{G \eta}(t)\right), \nabla \psi\right)_{\mathbb{L}^{2}(\Omega)^{d}}=(q(t), \psi)_{W}, \quad \forall \psi \in W, t \in[0, T] .
$$

We have the following result for Problem 4.6.

Lemma 4.7. There exists the unique solution $\varphi_{G \eta} \in W^{1.2}(0, T ; W)$ satisfying (4.8), moreover if $\varphi_{1}$ and $\varphi_{2}$ are two solutions to (4.8), then, there exists a constant $C>0$ such that

$$
\left|\varphi_{1}(t)-\varphi_{2}(t)\right|_{W} \leq C\left|u_{1}(t)-u_{2}(t)\right|_{V}, \forall t \in[0, T]
$$

Proof. Let $t \in[0, T]$, we use the Riesz-fréchet representation Theorem to define the operator $A_{G \eta}$ : $W \rightarrow W$ by

$$
\left(A_{G \eta}(t) \varphi, \psi\right)_{W}=\left(\beta \nabla \varphi_{G \eta}(t), \nabla \psi\right)_{\mathbb{L}^{2}(\Omega)^{d}}-\left(\xi \varepsilon\left(u_{G \eta}(t)\right), \nabla \psi\right)_{\mathbb{L}^{2}(\Omega)^{d}}, \quad \forall t \in[0, T] .
$$

For all $\varphi, \psi \in W$, let $\varphi_{1}, \varphi_{2} \in W$, then assumptions (3.6) and (3.7) imply

$$
\left(A_{G \eta}(t) \varphi_{1}-A_{G \eta}(t) \varphi_{1}, \varphi_{1}-\varphi_{2}\right)_{W} \geq m_{\beta}\left|\varphi_{1}-\varphi_{2}\right|_{W}^{2} .
$$

In other hand, from (3.7), it results

$$
\left(A_{G \eta}(t) \varphi_{1}-A_{G \eta}(t) \varphi_{1}, \psi\right)_{W} \leq c_{\beta}\left|\varphi_{1}-\varphi_{2}\right|_{W}|\psi|_{W},
$$

where $c_{\beta}$ is a positive constant which depends on $\beta$. Thus

$$
\left|A_{G \eta}(t) \varphi_{1}-A_{G \eta}(t) \varphi_{1}\right|_{W} \leq c_{\beta}\left|\varphi_{1}-\varphi_{2}\right|_{W} .
$$

Inequalities 4.11) and 4.12 show that the operator $A_{G \eta}(t)$ is a strongly monotone, Lipschitz continuous operator on $W$ and, therefore, there exists a unique element $\varphi_{G \eta}(t) \in W$ such that

$$
A_{G \eta} \varphi_{G \eta}(t)=q(t) .
$$


We combine 4.10) and 4.13) and find that $\varphi_{G \eta}(t) \in W$ is the unique solution of the nonlinear variational equation (4.8).

We show that $\varphi_{\xi \eta} \in W^{1.2}(0, T ; W)$. To this end, let $t_{1}, t_{2} \in[0, T]$ and, for the sake of simplicity, we write $\varphi_{G \eta}\left(t_{i}\right)=\varphi_{i}, u_{G \eta}\left(t_{i}\right)=u_{i}, q\left(t_{i}\right)=q_{i}$ for $i=1,2$.

It results from (3.6), 3.7), and (4.8)

$$
m_{\beta}\left|\varphi_{1}-\varphi_{2}\right|_{W}^{2} \leq c_{\xi}\left|u_{1}-u_{2}\right|_{V}\left|\varphi_{1}-\varphi_{2}\right|_{W}+\left|q_{1}-q_{2}\right|_{W}\left|\varphi_{1}-\varphi_{2}\right|_{W}
$$

where $c_{\xi}$ is a positive constant which depends on the piezoelectric tensor $\xi$.

$$
\left|\varphi_{1}(t)-\varphi_{2}(t)\right|_{W} \leq C\left(\left|u_{1}(t)-u_{2}(t)\right|_{V}+\left|q_{1}(t)-q_{2}(t)\right|_{W}\right) \forall t \in[0, T] .
$$

We also note that assumption 3.10$)$, combined with definition 3.11$)$ imply that $q \in \mathbb{L}^{2}(0, T ; W)$. Since $u_{G \eta} \in C^{1}(0, T ; K)$, inequality (4.15) implies that $\varphi_{G \eta} \in W^{1.2}(0, T ; W)$.

Let $\eta_{1}, \eta_{2} \in \mathbb{L}^{2}\left(0, T ; V^{\prime}\right), G_{1}, G_{2} \in \mathbb{L}^{2}\left(0, T ; \mathcal{H}_{1}\right)$ and let $\varphi_{G \eta}\left(t_{i}\right)=\varphi_{i}, u_{G \eta}\left(t_{i}\right)=u_{i}$, we use 4.8) and arguments similar to those used in the proof of (4.14) to obtain

$$
m_{\beta}\left|\varphi_{1}-\varphi_{2}\right|_{W} \leq c_{\xi}\left|u_{1}-u_{2}\right|_{V}+\left|q_{1}-q_{2}\right|_{W} .
$$

For all $t \in[0, T]$, this inequality leads to 4.9 which concludes the proof.

We consider the operator

$$
\begin{aligned}
\Lambda: \mathbb{L}^{2}\left(0, T ; \mathcal{H}_{1} \times V^{\prime}\right) & \rightarrow \mathbb{L}^{2}\left(0, T ; \mathcal{H}_{1} \times V^{\prime}\right) \text { be defined as } \\
\Lambda(G, \eta) & =\left(\Lambda_{1}(G), \Lambda_{2}(\eta)\right) \quad \forall G \in \mathbb{L}^{2}\left(0, T ; \mathcal{H}_{1}\right) \quad \forall \eta \in \mathbb{L}^{2}\left(0, T ; V^{\prime}\right), \\
\left|\Lambda\left(G_{1}, \eta_{1}\right)-\Lambda\left(G_{2}, \eta_{2}\right)\right|^{2} & =\left|\left(\Lambda_{1}\left(G_{1}\right), \Lambda_{2}\left(\eta_{1}\right)\right)-\left(\Lambda_{1}\left(G_{2}\right), \Lambda_{2}\left(\eta_{2}\right)\right)\right|^{2} \\
& =\left|\Lambda_{1}\left(G_{1}\right)-\Lambda_{1}\left(G_{2}\right)\right|^{2}+\left|\Lambda_{2}\left(\eta_{1}\right)-\Lambda_{2}\left(\eta_{2}\right)\right|^{2} .
\end{aligned}
$$

We show that $\Lambda$ has a unique fixed point.

\section{Lemma 4.8.}

$$
\Lambda\left(G^{*}, \eta^{*}\right)=\left(G^{*}, \eta^{*}\right) .
$$

Proof. Let $\left(G_{i}, \eta_{i}\right)$ be functions in $\mathbb{L}^{2}\left(0, T ; \mathcal{H}_{1} \times V^{\prime}\right)$ and denote by $\left(u_{i}, \varphi_{i}\right)$ the functions obtained in Lemmas 4.5 and 4.7, for $(G, \eta)=\left(G_{i}, \eta_{i}\right), i=1,2$. Let $t \in[0, T]$. We have

$$
\Lambda_{1}(G)=\mathcal{A} \varepsilon\left(\dot{u}_{G \eta}\right)+\mathcal{G} \varepsilon\left(u_{G \eta}\right)-\xi^{*} E\left(\varphi_{G \eta}\right) .
$$

So, we find

$$
\left|G_{1}(t)-G_{2}(t)\right|_{\mathcal{H}_{1}} \leq C\left(\left|v_{1}(t)-v_{2}(t)\right|_{V}+\left|u_{1}(t)-u_{2}(t)\right|_{V}+\left|\varphi_{1}(t)-\varphi_{2}(t)\right|_{W}\right) .
$$

Therefore 4.9 yields

$$
\left|G_{1}(t)-G_{2}(t)\right|_{\mathcal{H}_{1}} \leq C\left(\left|v_{1}(t)-v_{2}(t)\right|_{V}+\left|u_{1}(t)-u_{2}(t)\right|_{V}\right) .
$$

Using (4.5), we find

$$
\begin{array}{r}
\left(\mathcal{A} \varepsilon\left(v_{1}(t)\right)-\mathcal{A} \varepsilon\left(v_{2}(t)\right), v_{1}(t)-v_{2}(t)\right)+\left(\eta_{1}(t)-\eta_{2}(t), v_{1}(t)-v_{2}(t)\right) \\
+j\left(G_{1}, v_{1}(t)\right)-j\left(G_{1}, v_{2}(t)\right)-j\left(G_{2}, v_{1}(t)\right)+j\left(G_{2}, v_{2}(t)\right) \leq 0
\end{array}
$$


and, we have

$$
\begin{aligned}
j\left(G_{1}, v_{2}(t)\right)-j\left(G_{1}, v_{1}(t)\right)-j\left(G_{2}, v_{2}(t)\right)+j\left(G_{2}, v_{1}(t)\right) \\
=\int_{\Gamma_{3}} \mu p\left|R G_{1 \nu}\right|\left|v_{2 \tau}\right| d a-\int_{\Gamma_{3}} \mu p\left|R G_{1 \nu}\right|\left|v_{1 \tau}\right| d a \\
\quad-\int_{\Gamma_{3}} \mu p\left|R G_{2 \nu}\right|\left|v_{1 \tau}\right| d a+\int_{\Gamma_{3}} \mu p\left|R G_{2 \nu}\right|\left|v_{2 \tau}\right| d a .
\end{aligned}
$$

Moreover, from (3.3), (3.8), (3.13) and using the properties of $R$, we find

$$
j\left(G_{1}, v_{2}(t)\right)-j\left(G_{1}, v_{1}(t)\right)-j\left(G_{2}, v_{2}(t)\right)+j\left(G_{2}, v_{1}(t)\right) \leq C\left|G_{1}-G_{2}\right|_{\mathcal{H}_{1}}\left|v_{1}-v_{2}\right|_{V} .
$$

So, 4.18 will be

$$
\begin{gathered}
\left(\mathcal{A} \varepsilon\left(v_{1}(t)\right)-\mathcal{A} \varepsilon\left(v_{2}(t)\right), v_{1}(t)-v_{2}(t)\right)+\left(\eta_{1}(t)-\eta_{2}(t), v_{1}(t)-v_{2}(t)\right) \\
\leq C\left|G_{1}-G_{2}\right|_{\mathcal{H}_{1}}\left|v_{1}-v_{2}\right|_{V}
\end{gathered}
$$

We integrate this inequality with respect to time. We use the relation (3.4) and Cauchy-Schwartz's inequality to find that

$$
\begin{aligned}
m_{\mathcal{A}} \int_{0}^{t}\left|v_{1}(s)-v_{2}(s)\right|_{V}^{2} d s \leq \int_{0}^{t} & \left|\eta_{1}(s)-\eta_{2}(s)\right|_{V^{\prime}}\left|v_{1}(s)-v_{2}(s)\right|_{V} d s \\
& +C \int_{0}^{t}\left|G_{1}(s)-G_{2}(s)\right|_{\mathcal{H}_{1}}\left|v_{1}(s)-v_{2}(s)\right|_{V} d s
\end{aligned}
$$

for all $t \in[0, T]$. Then, using the inequality $2 a b \leq \frac{2 C}{m_{\mathcal{A}}} a^{2}+\frac{m_{\mathcal{A}}}{2 C} b^{2}$ and $2 a b \leq \frac{2}{m_{\mathcal{A}}} a^{2}+\frac{m_{\mathcal{A}}}{2} b^{2}$, we obtain

$$
\begin{aligned}
m_{\mathcal{A}} \int_{0}^{t}\left|v_{1}(s)-v_{2}(s)\right|_{V}^{2} d s \leq & \frac{1}{m_{\mathcal{A}}} \int_{0}^{t}\left|\eta_{1}(s)-\eta_{2}(s)\right|_{V^{\prime}}^{2} d s+\frac{m_{\mathcal{A}}}{4} \int_{0}^{t}\left|v_{1}(s)-v_{2}(s)\right|_{V}^{2} d s \\
& +\frac{C}{m_{\mathcal{A}}} \int_{0}^{t}\left|G_{1}(s)-G_{2}(s)\right|_{\mathcal{H}_{1}}^{2}+\frac{m_{\mathcal{A}}}{4} \int_{0}^{t}\left|v_{1}(s)-v_{2}(s)\right|_{V}^{2} d s .
\end{aligned}
$$

So, we find

$$
\int_{0}^{t}\left|v_{1}(s)-v_{2}(s)\right|_{V}^{2} d s \leq C\left(\int_{0}^{t}\left|\eta_{1}(s)-\eta_{2}(s)\right|_{V^{\prime}}^{2} d s+\int_{0}^{t}\left|G_{1}(s)-G_{2}(s)\right|_{\mathcal{H}_{1}}^{2} d s\right) .
$$

In other hand, since $u_{i}=u_{0}+\int_{0}^{t} v_{i}(s) d s$, we have

$$
\left|u_{1}(t)-u_{2}(t)\right|_{V}^{2} \leq C \int_{0}^{t}\left|v_{2}(s)-v_{1}(s)\right|_{V}^{2} d s .
$$

Applying this inequality in 4.20 we obtain

$$
\left|u_{1}(t)-u_{2}(t)\right|_{V}^{2} \leq C\left(\int_{0}^{t}\left|\eta_{1}(s)-\eta_{2}(s)\right|_{V^{\prime}}^{2} d s+\int_{0}^{t}\left|G_{1}(s)-G_{2}(s)\right|_{\mathcal{H}_{1}}^{2} d s\right) .
$$

Using (4.5), we find

$$
\begin{gathered}
\left(\mathcal{A} \varepsilon\left(v_{1}(t)\right)-\mathcal{A} \varepsilon\left(v_{2}(t)\right), v_{1}(t)-v_{2}(t)\right)+\left(\eta_{1}(t)-\eta_{2}(t), v_{1}(t)-v_{2}(t)\right) \\
+j\left(G_{1}, v_{1}(t)\right)-j\left(G_{1}, v_{2}(t)\right)-j\left(G_{2}, v_{1}(t)\right)+j\left(G_{2}, v_{2}(t)\right) \leq 0
\end{gathered}
$$


We use the relations (3.4), (4.19), and Cauchy-Schwartz's inequality to find that

$$
\begin{aligned}
m_{\mathcal{A}}\left|v_{1}(t)-v_{2}(t)\right|_{V}^{2} \leq & \left|\eta_{1}(t)-\eta_{2}(t)\right|_{V^{\prime}}\left|v_{1}(t)-v_{2}(t)\right|_{V} \\
& +C\left|G_{1}(t)-G_{2}(t)\right|_{\mathcal{H}_{1}}\left|v_{1}(t)-v_{2}(t)\right|_{V}
\end{aligned}
$$

for all $t \in[0, T]$. Then, using the inequalities $2 a b \leq \frac{2 C}{m_{\mathcal{A}}} a^{2}+\frac{m_{\mathcal{A}}}{2 C} b^{2}$ and $2 a b \leq \frac{2}{m_{\mathcal{A}}} a^{2}+\frac{m_{\mathcal{A}}}{2} b^{2}$, we obtain

$$
\begin{aligned}
m_{\mathcal{A}}\left|v_{1}(t)-v_{2}(t)\right|_{V}^{2} \leq & \frac{1}{m_{\mathcal{A}}}\left|\eta_{1}(t)-\eta_{2}(t)\right|_{V^{\prime}}^{2}+\frac{m_{\mathcal{A}}}{4}\left|v_{1}(t)-v_{2}(t)\right|_{V}^{2} \\
& +\frac{C}{m_{\mathcal{A}}}\left|G_{1}(t)-G_{2}(t)\right|_{\mathcal{H}_{1}}^{2}+\frac{m_{\mathcal{A}}}{4}\left|v_{1}(t)-v_{2}(t)\right|_{V}^{2} .
\end{aligned}
$$

So, we find

$$
\left|v_{1}(t)-v_{2}(t)\right|_{V}^{2} \leq C\left(\left|\eta_{1}(t)-\eta_{2}(t)\right|_{V^{\prime}}^{2}+\left|G_{1}(t)-G_{2}(t)\right|_{\mathcal{H}_{1}}^{2}\right) .
$$

We use the relations (4.21) and (4.22), to find that

$$
\begin{aligned}
\left|G_{1}(t)-G_{2}(t)\right|_{\mathcal{H}_{1}}^{2} \leq & C\left(\left|\eta_{1}(t)-\eta_{2}(t)\right|_{V^{\prime}}^{2}+\left|G_{1}(t)-G_{2}(t)\right|_{\mathcal{H}_{1}}^{2}\right) \\
& +C\left(\int_{0}^{t}\left|\eta_{1}(s)-\eta_{2}(s)\right|_{V^{\prime}}^{2} d s+\int_{0}^{t}\left|G_{1}(s)-G_{2}(s)\right|_{\mathcal{H}_{1}}^{2} d s\right) .
\end{aligned}
$$

In other hand

$$
\left(\Lambda_{2}(\eta), \chi\right)_{V^{\prime} \times V}=\left(\mathcal{G} \varepsilon\left(u_{G \eta}(t)+\xi^{*} \nabla \varphi_{G \eta}(t), \chi\right)_{V^{\prime} \times V}, \quad \forall \chi \in V .\right.
$$

We find

$$
\left|\eta_{1}(t)-\eta_{2}(t)\right|_{V^{\prime}}^{2} \leq C\left(\left|\varphi_{1}(t)-\varphi_{2}(t)\right|_{W}^{2}+\left|u_{1}(t)-u_{2}(t)\right|_{V}^{2}\right)
$$

Therefore, 4.9) yields

$$
\left|\eta_{1}(t)-\eta_{2}(t)\right|_{V^{\prime}}^{2} \leq C\left(\left|u_{1}(t)-u_{2}(t)\right|_{V}^{2}\right)
$$

From 4.21 we find

$$
\left|\eta_{1}(t)-\eta_{2}(t)\right|_{V^{\prime}}^{2} \leq C\left(\int_{0}^{t}\left|\eta_{1}(s)-\eta_{2}(s)\right|_{V^{\prime}}^{2} d s+\int_{0}^{t}\left|G_{1}(s)-G_{2}(s)\right|_{\mathcal{H}_{1}}^{2} d s\right) .
$$

Using 4.16), to see that

$$
\begin{aligned}
\left|\Lambda\left(G_{1}, \eta_{1}\right)-\Lambda\left(G_{2}, \eta_{2}\right)\right|_{\mathbb{L}^{2}\left(0 . T ; \mathcal{H}_{1} \times V^{\prime}\right)}^{2} \leq & C\left(\left|\eta_{1}(t)-\eta_{2}(t)\right|_{V^{\prime}}^{2}+\left|G_{1}(t)-G_{2}(t)\right|_{\mathcal{H}_{1}}^{2}\right) \\
& +C\left(\int_{0}^{t}\left|\eta_{1}(s)-\eta_{2}(s)\right|_{V^{\prime}}^{2} d s\right. \\
& \left.+\int_{0}^{t}\left|G_{1}(s)-G_{2}(s)\right|_{\mathcal{H}_{1}}^{2} d s\right)
\end{aligned}
$$

for all $t \in[0, T]$, denoting by $p$ the powers of operator $\Lambda$, 4.23 implies by recurrence that

$$
\begin{aligned}
\left|\Lambda^{p}\left(G_{1}, \eta_{1}\right)-\Lambda^{p}\left(G_{2}, \eta_{2}\right)\right|_{\mathbb{L}^{2}\left(0, T ; \mathcal{H}_{1} \times V^{\prime}\right)}^{2} & \leq\left(\sum_{j=0}^{p} C_{p}^{j} C^{p-j} \frac{C^{j} T^{j}}{j !}\right)\left|\left(G_{1}, \eta_{1}\right)-\left(G_{2}, \eta_{2}\right)\right|_{\mathbb{L}^{2}\left(0, T ; \mathcal{H}_{1} \times V^{\prime}\right)}^{2} \\
& \leq \frac{(C p+C T)^{p}}{p !}\left|\left(G_{1}, \eta_{1}\right)-\left(G_{2}, \eta_{2}\right)\right|_{\mathbb{L}^{2}\left(0, T ; \mathcal{H}_{1} \times V^{\prime}\right)}^{2} .
\end{aligned}
$$


Using the Stirling's formula, we obtain under the condition $C \leq \frac{1}{e}$ that

$$
\lim _{p \rightarrow \infty} \frac{(C p+C T)^{p}}{p !}=0
$$

which shows that for a sufficiently large $p$ the operator $\Lambda^{p}$ is a contraction on the Banach space $\mathbb{L}^{2}\left(0, T ; \mathcal{H}_{1} \times V^{\prime}\right)$ and therefore, there exists a unique element $\left(G^{*}, \eta^{*}\right) \in \mathbb{L}^{2}\left(0, T ; \mathcal{H}_{1} \times V^{\prime}\right)$ such that

$$
\Lambda\left(G^{*}, \eta^{*}\right)=\left(G^{*}, \eta^{*}\right)
$$

From 4.17), we find

$$
\left(G^{*}, \eta^{*}\right)=\left(\sigma_{G^{*} \eta^{*}}, \xi^{*} \nabla \varphi_{G^{*} \eta^{*}}+\mathcal{G} \varepsilon\left(u_{G^{*} \eta^{*}}\right)\right)
$$

Now, we have all the ingredients to provide the proof of Theorem 4.1 .

Proof of Theorem 4.1.

Existence. Let $\left(G^{*}, \eta^{*}\right) \in \mathbb{L}^{2}\left(0, T ; \mathcal{H}_{1} \times V^{\prime}\right)$ be the fixed point of $P V 1_{G \eta}$ and let $\left(u^{*}, \varphi^{*}\right)$ be the solution to Problems 4.2 and 4.6 for $(G, \eta)=\left(G^{*}, \eta^{*}\right)$, that is, $u^{*}=u_{G^{*} \eta^{*}}$ and $\varphi^{*}=\varphi_{G^{*} \eta^{*}}$. It results from (3.15) and (3.16) that $\left(u^{*}, \varphi^{*}\right)$ is a solution of Problem 3.1. Properties (4.1) and (4.2) follow from Lemmas 4.5 and 4.7 .

Uniqueness. The uniqueness of the solution is a consequence of the uniqueness of the fixed point of operator defined by 4.16 .

\section{References}

[1] R. C. Batra, J. S. Yang, Saint-Venant's principle in linear piezoelectricity, J. Elasticity, 38 (1995), 209-218. 1

[2] P. Bisegna, F. Lebon, F. Maceri, The unilateral frictional contact of a piezoelectric body with a rigid support, Contact mechanics, Praia da Consolação, (2001), 347-354, Solid Mech. Appl., Kluwer Acad. Publ., Dordrecht, (2002). 1

[3] A. Capatina, Inéquations variationnelles et problémes de contact avec frottement, Preprint Series IMAR, 10 (2011), 1-210. 4.4

[4] T. Ikeda, Fundamentals of piezoelectricity, Oxford university press, Oxford, (1990). 1

[5] F. Maceri, P. Bisegna, The unilateral frictionless contact of a piezoelectric body with a rigid support, Recent advances in contact mechanics, Math. Comput. Modelling, 28 (1998), 19-28. 1

[6] R. D. Mindlin, Polarization gradient in elastic dielectrics, Int. J. Solids Struct., 4 (1968), 637-642. 1

[7] R. D. Mindlin, Elasticity, piezoelectricity and crystal lattice dynamics, J. Elasticity, 4 (1972), 217-282. 1

[8] V. Z. Patron, B. A. Kudryavtsev, Electromagnetoelasticity, piezoelectrics and electrically conductive solids, Gordon \& Breach Science Publishers, New York, (1988). 1

[9] M. Sofonea, E. H. Essoufi, A piezoelectric contact problem with slip dependent coefficient of friction, Math. Model. Anal., 9 (2004), 229-242. 1

[10] M. Sofonea, E. H. Essoufi, Quasistatic frictional contact of a viscoelastic piezoelectric body, Adv. Math. Sci. Appl., 14 (2004), 613-631. 1

[11] M. Sofonea, W. M. Han, M. Shillor, Analysis and approximation of contact problems with adhesion or damage, Pure and Applied Mathematics (Boca Raton), Chapman \& Hall/CRC, Boca Raton, FL, (2006). 1

[12] N. Strömberg, L. Johansson, A. Klarbring, Derivation and analysis of a generalized standard model for contact friction and wear, Int. J. Solids Struct., 33 (1996), 1817-1836. 1 